is negative for sufficiently small positive values of $z$, it follows that $F(z)$ decreases from the value zero to a certain negative minimum value, as $z$ increases from 0 to $z_{0}$; then as $z$ increases

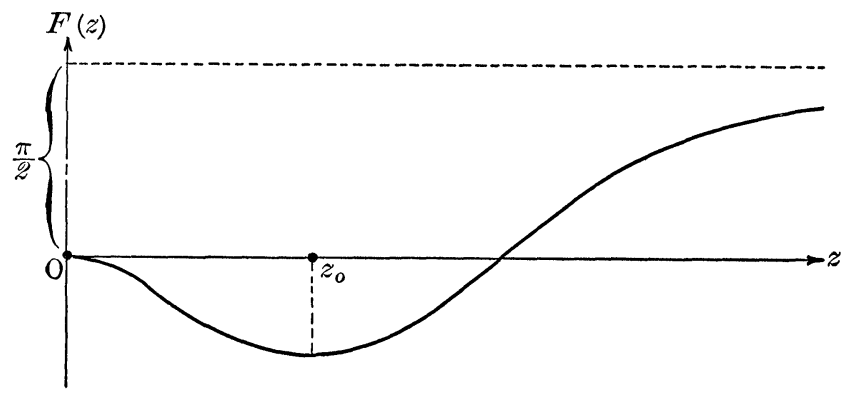

from $z_{0}$ to $+\infty, F(z)$ increases continually and approaches for $z=+\infty$ the limit $+\pi / 2$. Hence the equation (12) has one and but one real positive root.

This root being found, the equations (11), (10), and (9) yield a unique solution $\omega_{0}, \omega_{1}$, of (7) and (8), satisfying the inequality (4). Finally the values of $h$ are $z$ follow unambiguously from (3).

The existence and uniqueness of the solution of the proposed problem are therefore proved.

UNIVERSITY OF Chicago,

November 2, 1903.

\title{
ON THREE TYPES OF SURFACES OF THE THIRD ORDER REGARDED AS DOUBLE SURFACES OF TRANSLATION.
}

BY DR. A. S. GALE.

(Read before the American Mathematical Society, October 31, 1903.)

THIS note serves the double purpose of making a slight addition to the theory of three types of surfaces of the third order and of exhibiting the double surfaces of translation of lowest order. The latter surfaces enjoy all the properties of the double minimum surfaces * except those immediately dependent on the

* Lie, Math. Annalen, vol. 14 (1879), p. 346 et seq. ; Darboux, Théorie des surfaces, vol. 1, p. 348 et seq. 
minimum property; and as Henneberg's minimum surface of order fifteen is the simplest of the real double minimum surfaces, it is not uninteresting to see what very simple surfaces have many of the same properties.

By a double surface of translation is meant a surface whose equations have the form

$S: \quad x=A(u)+A(v), \quad y=B(u)+B(v), \quad z=C(u)+C(v)$

Such a surface may be generated in either one of two ways $1^{\circ}$ by a curve

$C: \quad x=A(u), \quad y=B(u), \quad z=C(u)$,

subjected to the one-parameter system of translations

$$
x^{\prime}=x+A(v), \quad y^{\prime}=y+B(v), \quad z^{\prime}=z+C(v) ;
$$

$2^{\circ}$ by the middle point of a chord of the curve

$$
x=2 A(u), \quad y=2 B(u), \quad z=2 C(u) .
$$

If the surface $S$ be subjected to a projective transformation, the transformed surface may evidently be regarded in two ways: $1^{\circ}$ as the locus of a curve subjected to a one-parameter system of projective transformations of which the invariant configurations are surface elements with a common plane; $2^{\circ}$ as the locus of the fourth harmonic to two points of intersection of a secant of a curve $C$ and the point in which that secant meets a fixed plane.

Since two surfaces of the third order belong to the same type if one may be projected into the other, it is evident that these projective definitions will afford means of generating any surface of the third order if a surface of the same type may be regarded as a double surface of translation. The surface $S$ will be of the third order, by Lie's theorem on the order of surfaces of translation,* if $C$ is a twisted cubic ; and if the axes be suitably chosen the elimination of $u$ and $v$ presents no difficulty, thus giving the equation of $S$ in rectangular coördinates from which the nature of the singularities of $S$ may be readily determined.

We find in this way that, if $C$ cuts the plane at infinity in three distinct points $P, Q$ and $R$, then $S$ will be a surface of

\footnotetext{
* L. c., pp. 359, 354; see also Darboux, l. a, p. 369.
} 
the third order with three conical points, $C_{2}$, situated at $P, Q$ and $R$. If, however, $C$ cuts the plane at infinity at $P$ and is tangent to it at $Q$ then the only singularities of $S$ are a conical point $C_{2}$ at $P$ and a binode $B_{5}$ at $Q$ whose axis lies on $S$, one of the biplanes being tangent to $S$. Finally, if the plane at infinity is an osculating plane of $C$, then $S$ is Cayley's ruled surface $*$ of the third order.

The second projective definition then affords a simple means of generating any surface of these three types. When, however, $C$ is a twisted cubic, that definition declares that $S$ is the transformed of a plane by the involutory cubic transformation of space under which corresponding points lie on a secant of $C$ and are harmonically situated with respect to the points of intersection of that secant with $C$. The above results have already been given from this point of view $\dagger$ but attention does not seem to have been called to the fact that the surface corresponding to the plane at infinity is a surface of translation.

Remarking that a projective transformation transforms a conjugate system of curves on a surface into a conjugate system on the transformed surface, the properties of double surfaces of translation afford the following theorem :

On the surfaces of the third order whose singularities are three conical points, or a conical point and a binode whose axis lies on the surface, one of the biplanes being tangent to the surface, and on Cayley's ruled surface of the third order, there is a singly infinite system of twisted cubics any one of which may be transformed into any other by a projective transformation of a system similar to a system of translations under a projective transformation $T$. Two of these cubics pass through each point of general position on the surface. These cubics form a conjugate system ; they have an envelope into which any one may be transformed by a projective transformation similar under $T$ to a homothetic transformation whose ratio is two. This envelope is the locus of points through which but one curve of the system passes and is an asymptotic line of the surface. The surface may be regarded as the locus

* Lie, 1. c., footnote, p. 353.

†Cantonne, Napoli Rendiconti, vol. 25 (1886), pp. 186, 189 and 190. The transformation is studied by Reye, Geometrie der Lage, 3d ed., part 3, p. 129 and Schoute, Nieuw Archief, 2d series vol. 4 (1899). If we consider the transformation in which the cubic is replaced by any twisted curve, Lie's theorem on the order of a surface of translation affords the order of the surface corresponding to a plane. 
of the fourth harmonic to two points of this envelope and the point of intersection of the secant through these two points with the plane of the singularities of the surface.

YALE UnIVERSITY, October, 1903.

\section{ON THE GENERATION OF FINITE FROM INFIN- ITESIMAL TRANSFORMATIONS - A CORRECTION.}

BY PROFESSOR H. B. NEWSON.

(Read before the Chicago Section of the American Mathematical Society, January 2, 1903.)

IN a paper entitled "Continuous groups of circular transformations" which the author read before this Society, April 24, 1897, and which was published in the Bulcetrs (2) series, volume 4, pages 107-121, there occurs a serious error * which I desire to correct.

The error in question is a misstatement of the number of logarithmic spirals of the family $\rho=e^{(c+i) \theta}$ (where $c$ is a parameter) that pass through a given point of the plane. It was stated on page 114 of the above mentioned paper that in general only two spirals of the family pass through a given point. In fact there are an infinite number of these spirals through a point $P$.

To show this let, the coördinates of $P$ be $\left(\rho_{1}, \theta_{1}+2 n \pi\right)$. Since $\rho_{1}=e^{(c+i)\left(\theta_{1}+2 n \pi\right)}$, then

$$
\log \rho_{1} \equiv \log r+2 i m \pi=c\left(\theta_{1}+2 n \pi\right)+i\left(\theta_{1}+2 n \pi\right) ;
$$

whence $\log r=c\left(\theta_{1}+2 n \pi\right)$ or $c=\log r /\left(\theta_{1}+2 n \pi\right)$. Since $n$ is any integer, $c$ may have any one of an infinite number of values. Thus there are an infinite number of spirals of the family through the point $\left(\rho_{1}, \theta_{1}+2 n \pi\right)$. When $n=0,1,2$, $3, \ldots$ the corresponding spiral, starting from the origin, makes $0,1,2,3, \ldots$ turns about the origin before passing through the point $P$.

The last paragraph on page 114 and the first on page 115 , including theorems 7 and 8 , of the above-mentioned article should be corrected to read as follows :

* My attention was first called to this error by Professor Frank Morley. 